\title{
Changing Salinity Affects Hematological and Histological Response in Hybrids and Hybrid Triploids between River Puffer, Takifugu obscurus and Tiger Puffer, T. rubripes
}

\author{
${ }^{\dagger}$ In-Seok Park ${ }^{1}$, Hyun Woo Gil ${ }^{1}$, and Ji Su Oh${ }^{2}$ \\ ${ }^{1}$ Division of Marine Bioscience, College of Ocean Science and Technology, \\ Korea Maritime \& Ocean University, Busan 49112, Korea \\ ${ }^{2}$ National Agency for Marine Seed Improvement, National Institute of Fisheries Science, Busan 46083, Korea
}

\begin{abstract}
We investigated the physiological responses of the river puffer, Takifugu obscurus, the tiger puffer, T. rubripes, a hybrids of the two (river puffer $\times$ tiger puffer) and a hybrid triploids to acute changes of salinity from $30 \mathrm{psu}$ to $0 \mathrm{psu}$ and from $0 \mathrm{psu}$ to $30 \mathrm{psu}$. The blood and plasma factors of each species were elevated for 48, 72, or $96 \mathrm{hrs}$, and thereafter decreased due to hyper-osmoregulation and hypo-osmoregulation. In hyper-osmoregulation and hypo-osmoregulation, the cortisol concentration of river puffer, hybrids, hybrid triploids and tiger puffer increased for 12 or 48 hrs, and decreased thereafter. Chloride cells in the gill filaments of each species increased with increasing salinity, and melano-macrophages in the kidney tissue of each species increased with decreasing salinity. In conclusion, the hematological and stress responses of the hybrids were between those of the river puffer and tiger puffer, and the hematological responses of the hybrid triploids were higher than those of the other groups. The stress response of the hybrids was more sensitive than that of the hybrid triploids. In all groups, the histological responses of kidney in hyper-osmoregulation were more sensitive than those in hypo-osmoregulation.

Key words : Hematology, Histology, Hybrids, Hybrid triploids, Osmoregulation, River puffer, Tiger puffer
\end{abstract}

\section{INTRODUCTION}

Domestic consumption of puffer fish is around 10,000 tons per year in Korea. Most of domestic puffer fish is imported from other countries such as China and puffer fish is regarded as high value added fish in Japan and Korea (Kang et al., 2007). River puffer, Takifugu obscurus has better taste than other puffer fish, anti-cancer effect and superior in detoxification (Jeong et al., 1994; Kim et al., 2006). But river puffer is not suitable to breed because breeding method is very difficult and takes more 30 months to matured fish. River puffer and tiger puffer, $T$. rubripes taxonomicly are defined Tetraodontiformes, Tetraodontidae. Tiger puffer inhabit the coast such as Korea and China, but river puffer inhabit river (in fry stage), brackish water zone (in juvenile), and coast (in adult) (Jeong et al., 1994; Kim et al., 2006; Kang et al., 2007). River puffer migrate to river for spawning (Jeong et al., 1994; Kim et al., 2006; Kang et al., 2007). River puffer

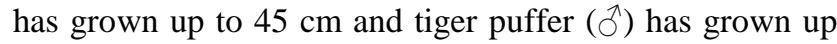
to $75 \mathrm{~cm}$ and river puffer and tiger puffer feed are shrimp, small fish and etc. (Park et al., 1997; Kikuchi et al., 2007).

\footnotetext{
Manuscript received November 22, 2018, Received in revised form January 7, 2019, Accepted June 10, 2019

${ }^{\dagger}$ Corresponding Author: In-Seok Park, Division of Marine Bioscience, College of Ocean Science and Technology, Korea Maritime \& Ocean University, Busan 49112, Korea. Tel: +82-51-410-4321, Fax: +82-51-404-4750, E-mail: ispark@kmou.ac.kr
}

This is an Open Access article distributed under the terms of the Creative Commons Attribution Non-Commercial License (http:// creative-commons.org/licenses/by-nc/3.0) which permits unrestricted non-commercial use, distribution, and reproduction in any medium, provided the original work is properly cited. 
Puffer fish has a distinct trait which is strong poison, tetradotoxin. Toxic exists in ovary, liver, intestines, but toxic is varied in breeding, seasonal, object-specific, and region (Jeong et al., 1994; Kim et al., 2006).

With interspecific hybrids, there are many variables in early survival rate, hatched rate and abnormal rate in fish (Chevassus, 1983; Yoo et al., 2018). Heterosis means artificially induced offspring hybrids fish dominant to parents fish and is considered strong in sickness and resistant to change (Chevassus, 1983; Kim et al., 1995; Park \& Kim, 2000; Bryden et al., 2004; Rahman et al., 2005; Yoo et al., 2018). Hybrids also have better feed conversion; tolerance to low oxygen; increased resistance to many diseases; high sulfide concentration, low ambient temperature and water of various $\mathrm{pH}$ value tolerance (Chevassus, 1983; Kim et al., 1995; Bryden et al., 2004; Rahman et al., 2005). Besides, the flavor of hybrids is considered to be equivalent to that of the puffer fish, definitely much better than the tiger puffer (Yoo \& Bai, 2014).

The induction of triploids in fish has been used to generate sterility for applications in commercial farming and fishery management (Benfey, 1999). Triploids have impaired gametogenesis, and investment in somatic growth may not be hindered by the metabolic costs of sexual maturation. Additionally, sterility in triploids may be used to prevent the decline in flesh quality associated with sexual maturation, and also removes concerns regarding the environmental impact of farmed escapees (Peruzzi et al., 2004). The methods used to induce triploids, the performance of triploids, and the rationale for their practical use have been reviewed (Park \& Kim, 2000; Seol et al., 2008). By conferring sterility to exotic fish for a limited purpose, triploids can serve as an effective method to reduce or eliminate the environmental risks associated with genetically modified organisms (Kim et al., 1994). Various fish species has been induced to triploids by cold or heat shock, including ayu, Plecoglossus altivelis, cherry salmon, Oncorhynchus masou, European sea bass, Dicentrarchus labrax, Far Eastern catfish, Silurus asotus, rainbow trout, O. mykiss, river puffer, and tiger puffer (Benfey, 1999; Seol et al., 2008 ; Park et al., 2017).

Numerous studies concerning the growth of triploids have been published. Growth tends to slow or cease in maturing fish, and it is during the later stages of sexual maturation that a growth advantage of triploids over diploids is most likely to be observed (Benfey, 1999). In species that survive spawning, diploids frequently exhibit compensatory growth and may overcome any disadvantage (Benfey, 1999). Therefore, a growth advantage of triploids is most likely to be seen in species where the diploids have high or complete mortality associated with sexual maturation and spawning, as in ayu and twice-spawned rainbow trout (Benfey, 1999).

Changes in the salinity induced changes of osmoregulation. When water salinity changed in the osmoregulation, the fish remain constant to the body moisture and intracellular salts and should try to regulate the concentration of nutritional substances. The osmoregulation regulator of bony fish is in gill, gut and kidney. When salinity changes, fish should adapt osmoregulation and works with direct stress factors (Morgan \& Iwama, 1991).

River puffer taxonomically defined Tetraodontiformes, Tetraodontidae, inhabit the coast in Korea and China, going up to the upstream in the middle of river in spawning season (Kikuchi et al., 2007; Park et al., 2017; Park, 2019). Therefore, determination of physiological response of river puffer, tiger puffer, hybrids between river puffer $(q)$ and tiger puffer $(\lesssim)$, and hybrid triploids under environment of changing salinity is necessary for determining characteristics of hybrids and hybrid triploids. Methods for determine physiological response of hybrids and hybrid triploids are hematological analysis and histological observation of gill and kidney and measurement of cortisol concentration. Therefore, the purpose of this study is to describe physiological responses of the blood and plasma factors in hybrids between river puffer $(+)$ and tiger puffer $(\circlearrowleft)$, and the hybrid 
triploids, and conform histological change of hybrids and hybrid triploids under hyper-osmoregulation and hypoosmoregulation .

\section{MATERIALS AND METHODS}

On March 20 2016, 50 river puffer, Takifugu obscurus $(\bigcirc)$ and tiger puffer, T. rubripes $(\lesssim)$ obtained from puffer fish hatchery, Korea. During experimental period, the fish were reared for this experiment at the Fishery Genetics and Breeding Sciences Laboratory of the Korea Maritime \& Ocean University, Busan, Korea. Experiments fish had an under these conditions, reared in an aquarium maintained at a temperature of $20 \pm 1.5^{\circ} \mathrm{C}$. Feed is that experimental fish had a commercial feed (Cheonhajaeil Feed Corporation, Korea) twice a day.

On April 8 2016, anaesthetized 30 female river puffer and 30 male tiger puffer with clove oil and lidocaine- $\mathrm{HCl}$ (Park, 2019) were injected human chorionic gonadotropin, HCG (Sigma, USA) 1,000 IU/kg BW, respectively. 24 hrs after HCG injection, extracted eggs of river puffer and sperms of tiger puffer were fertilized using the method of
Park et al. (2017) and Yoo et al. (2018). A total of 3,000 fertilized eggs were subjected to cold-shock treatment $\left(4^{\circ} \mathrm{C}\right)$ to prevent extrusion of the second polar body (Park et al., 2017). Untreated fertilized eggs were used as hybrids groups. During 5 months before this experiment, samples were reared and fed two times in everyday, and water temperature and salinity were maintained $25 \pm 1.0^{\circ} \mathrm{C}$ and $30 \pm 0.5 \mathrm{psu}$ by aquarium maintenance system. Using the method of Yoo et al. (2018), a flowcytometric analysis was performed to estimate the average celluar DNA content of 100 individuals from each species. Fin tissues were collected from caudal fin of experimental samples. Samples were fixed in $10 \mathrm{~mL}$ of cold $70 \%$ ethanol. Ethanol fixed one million cells from caudal fin tissues were collected and stained using a high-resolution DNA staining kit (Partec $\mathrm{GmbH}$, Germany) under dark conditions about $15 \mathrm{~min}$. Stained samples were analyzed on Partec PA-II flowcytometry (Partec GmbH, Germany) to distinction of hybrids and hybrid triploids.

Each experiment used 50 experimental fishes and set triplicate. Table 1 showed water quality items of 0 psu, 15 psu, and 30 psu sea water. Before experiment, experimen-

Table 1. Water quality items for this experiment in river puffer, Takifugu obscurus, tiger puffer, T. rubripes, hybrids (river puffer $q \times$ tiger puffer $\circlearrowleft$ ), and hybrid triploids*

\begin{tabular}{cccc}
\hline \hline Test parameters & \multicolumn{3}{c}{ Condition } \\
\cline { 2 - 4 } & $0 \mathrm{psu}$ & $15 \mathrm{psu}$ & $30 \mathrm{psu}$ \\
\hline Temperature $\left({ }^{\circ} \mathrm{C}\right)$ & $26 \pm 0.5$ & $26 \pm 0.5$ & $26 \pm 0.5$ \\
$\mathrm{pH}$ & $7.1 \pm 0.65$ & $7.1 \pm 0.78$ & $7.1 \pm 1.02$ \\
DO (dissolved oxygen: $\mathrm{mg} / \mathrm{L} ;$ & 7.6 & 7.5 & 7.5 \\
saturated concentration in $\left.25^{\circ} \mathrm{C}\right)$ & 0.01 & 0.06 & 0.06 \\
Ammonia $(\mathrm{ppm})$ & $1.8 \pm 0.14$ & $1.8 \pm 0.12$ & $1.7 \pm 0.19$ \\
Nitric acid (ppm) & 0.01 & 0.01 & 0.02 \\
Nitrous acid (ppm) & & & \\
\hline
\end{tabular}

*Temperature, $\mathrm{pH}$, dissolved oxygen and salinity were measured using an oxygen measurement electrode and a multi-data logger system (Oxyguard, Denmark). Ammonia, nitric acid and nitrous acid were measured using spectrophotometer (DR2800, HACH, Loveland, Colorado, USA). The values are means of triplicate groups $(\mathrm{n}=50)$. 
tal fishes were adapted 0 psu and 30 psu during $168 \mathrm{hrs}$, respectively, and experimental water were exchanged daily. The kidney and gill tisues were removed from 5 samples of each species for histological observation. Hyper-osmoregulation decreased 15 psu per a day from 30 psu to 0 psu, and hypo-osmoregulation increased 15 psu per a day from 0 psu to $30 \mathrm{psu}$. While exchanging water, samples prevented that exposure to air for minimize stress response of other factors. After experiment, blood samples from caudal vein of each sample were extracted from 50 randomly selected fish at 0 (pre), 24, 48, 72, 96, and $120 \mathrm{hrs}$ after changing salinity, and the kidney and gill tisues were removed from 5 samples of each species after experiment.

Hematological analysis was measured by auto hematology analyzer (PE-6800, Prokan, China) with fresh sample blood (Park et al., 2017). After hematological analysis, blood was centrifuged at $200 \times \mathrm{g}$ for $10 \mathrm{~min}$ and stored at $-80^{\circ} \mathrm{C}$ freezer (Nihon Freezer, Japan) until analysis. Blood samples were measured cortisol concentration using radioimmunoassay. Cortisol was determined in $50 \mu \mathrm{L}$ samples using RIA kits (Coat-A-Count TKCO Cortisol RIA Kit; DPC, USA). Mixtures of sample in $100 \mathrm{~mL}$ antiserum were incubated for $45 \mathrm{~min}$ at $37^{\circ} \mathrm{C}$, and then $1,000 \mathrm{~mL}$ separation reagent was added. The mixture was placed in a refrigerator at $4^{\circ} \mathrm{C}$ for $15 \mathrm{~min}$ and then centrifuged at 1,200 $\mathrm{g}$ for $15 \mathrm{~min}$. Supernatant was assayed for gamma radiation using an automatic gamma counter (Cobra; Packard, USA).

Extracted kidney and gill tissues were fixed in $10 \%$ neutral formation solution (100 mL, formalin, $6.5 \mathrm{~g} \mathrm{Na}_{2} \mathrm{HPO}_{4} \cdot 12 \mathrm{H}_{2} \mathrm{O}$, $4.5 \mathrm{~g} \mathrm{KH}_{2} \mathrm{PO}_{4}, 900 \mathrm{~mL} \mathrm{DW}$ ) for $24 \mathrm{hrs}$. The fixed samples were then refixed in Bouin's solution for a further $24 \mathrm{hrs}$. All fixed tissue were routinely dehydrated in ethanol, equilibrated in xylene, and embedded in paraffin according to standard histological techniques. Cross sections were then cut at $6 \mu \mathrm{m}$ and routinely stained using Mayer's Haematoxlyin and eosin Y-phroxine B before being observed under a high-powered microscope (Carl Zeiss, Germany). Using the SPSS statistics package (SPSS 12.0, SPSS Inc.,
Chicago, IL, USA), one-way analysis of variance (ANOVA) were carried out to test for statistical significance between diploid and triploid fish. Multiple comparisons were performed using Duncan's multiple range test $(p<0.05$; Duncan, 1955).

\section{RESULTS}

\section{Hematological response}

Table 2 shows the blood and plasma factors during the hyper-osmoregulation of salinity. The blood and plasma factors of the river puffer, Takifugu obscurus, the hybrids, hybrid triploids, and tiger puffer, $T$. rubripes, were elevated for 48,72 , or $96 \mathrm{hrs}$, and decreased thereafter. The hematocrit values of the river puffer, the hybrids and hybrid triploids after $120 \mathrm{hrs}$ were higher than those at $0 \mathrm{hrs}(p<0.05)$. However, the hematocrit value of the tiger puffer after 120 hrs was lower than that at $0 \mathrm{hr}(p<0.05)$. At $0 \mathrm{hr}$, the hematocrit value of the tiger puffer was higher than those of the other groups, and the hematocrit value of the hybrid was the lowest $(p<0.05)$. At $120 \mathrm{hrs}$, the hematocrit value of the tiger puffer was lower than those of the other groups, and the hematocrit value of the hybrid triploids was the highest $(p<0.05)$. In addition, the hematocrit value of the hybrids was higher than that of the tiger puffer $(p<0.05)$.

The hemoglobin values of each species at $120 \mathrm{hrs}$ were higher than those at $0 \mathrm{hr}(p<0.05)$. At $0 \mathrm{hr}$, the hemoglobin value of the tiger puffer was higher than those of the other groups, while the hemoglobin value of the river puffer was the lowest $(p<0.05)$. At 120 hrs, the hemoglobin value of the hybrids was lower than those of the other groups and the hemoglobin value of the hybrid triploids was the highest $(p<0.05)$. The red blood cells of the hybrids and hybrid triploids at $120 \mathrm{hrs}$ were higher than those at $0 \mathrm{hr}$ $(p<0.05)$. At $0 \mathrm{hr}$, the red blood cells of the tiger puffer were higher than those of the other groups, while the red blood cells of the hybrid triploids were the lowest $(p<0.05)$. 
Table 2. Comparison of blood and plasma factors during $120 \mathrm{hrs}$ after hyper-osmoregulation of salinity in river puffer, Takifugu obscurus, tiger puffer, T. rubripes, hybrids (river puffer $q \times$ tiger puffer ${ }^{\lambda}$ ), and hybrid triploids*

\begin{tabular}{|c|c|c|c|c|c|}
\hline \multirow{2}{*}{ Items } & \multirow{2}{*}{$\begin{array}{c}\text { Elapsed time } \\
\text { (hrs) }\end{array}$} & \multicolumn{4}{|c|}{ Experiment groups } \\
\hline & & River puffer & Hybrids & Hybrid triploids & Tiger puffer \\
\hline \multirow{6}{*}{$\begin{array}{c}\text { Hematocrit } \\
(\%)\end{array}$} & 0 & $24.4 \pm 4.9^{\mathrm{a}}$ & $24.3 \pm 5.9^{\mathrm{a}}$ & $25.4 \pm 4.1^{b}$ & $28.4 \pm 5.1^{\mathrm{a}}$ \\
\hline & 24 & $32.5 \pm 3.2^{\mathrm{ab}}$ & $41.8 \pm 8.6^{\mathrm{b}}$ & $24.1 \pm 7.2^{\mathrm{a}}$ & $35.5 \pm 3.9^{\mathrm{ab}}$ \\
\hline & 48 & $38.9 \pm 7.4^{\mathrm{b}}$ & $40.0 \pm 6.5^{\mathrm{b}}$ & $43.2 \pm 5.5^{\mathrm{c}}$ & $38.0 \pm 8.1^{\mathrm{b}}$ \\
\hline & 72 & $31.0 \pm 5.6^{\mathrm{ab}}$ & $28.6 \pm 9.0^{\mathrm{a}}$ & $38.4 \pm 6.4^{b}$ & $31.4 \pm 6.0^{\mathrm{ab}}$ \\
\hline & 96 & $31.8 \pm 7.4^{\mathrm{a}}$ & $35.3 \pm 7.2^{\mathrm{ab}}$ & $40.7 \pm 9.7^{\mathrm{bc}}$ & $31.0 \pm 5.1^{\mathrm{ab}}$ \\
\hline & 120 & $25.8 \pm 3.0^{\mathrm{a}}$ & $29.1 \pm 3.3^{\mathrm{ab}}$ & $36.6 \pm 3.4^{\mathrm{bc}}$ & $24.8 \pm 2.4^{\mathrm{a}}$ \\
\hline \multirow{6}{*}{$\begin{array}{l}\text { Hemoglobin } \\
(\mathrm{g} / \mathrm{dL})\end{array}$} & 0 & $9.8 \pm 0.3^{\mathrm{a}}$ & $10.1 \pm 0.6^{a}$ & $10.1 \pm 0.7^{\mathrm{a}}$ & $10.8 \pm 1.0^{\mathrm{a}}$ \\
\hline & 24 & $12.8 \pm 0.8^{\mathrm{ab}}$ & $14.8 \pm 0.4^{\mathrm{ab}}$ & $11.1 \pm 3.0^{\mathrm{a}}$ & $12.1 \pm 0.9^{\mathrm{ab}}$ \\
\hline & 48 & $14.5 \pm 2.3^{b}$ & $17.5 \pm 4.1^{\mathrm{b}}$ & $15.8 \pm 0.6^{\mathrm{b}}$ & $15.5 \pm 3.1^{\mathrm{b}}$ \\
\hline & 72 & $14.3 \pm 2.3^{b}$ & $14.9 \pm 3.4^{\mathrm{ab}}$ & $14.5 \pm 1.3^{\mathrm{b}}$ & $14.8 \pm 2.8^{\mathrm{b}}$ \\
\hline & 96 & $11.0 \pm 1.3^{\mathrm{ab}}$ & $13.0 \pm 1.3^{\mathrm{ab}}$ & $14.4 \pm 0.9^{b}$ & $12.4 \pm 1.5^{\mathrm{ab}}$ \\
\hline & 120 & $12.4 \pm 0.7^{\mathrm{ab}}$ & $10.6 \pm 3.9^{a}$ & $14.7 \pm 0.1^{b}$ & $11.0 \pm 1.1^{\mathrm{ab}}$ \\
\hline \multirow{6}{*}{$\begin{array}{l}\text { Red blood cell } \\
\left(\times 10^{6} \text { cell } / \mu \mathrm{L}\right)\end{array}$} & 0 & $2.7 \pm 0.7^{\mathrm{a}}$ & $2.8 \pm 0.5^{\mathrm{a}}$ & $2.6 \pm 0.5^{\mathrm{ab}}$ & $2.9 \pm 1.1^{\mathrm{a}}$ \\
\hline & 24 & $3.6 \pm 0.1^{\mathrm{ab}}$ & $4.3 \pm 0.3^{\mathrm{b}}$ & $2.2 \pm 0.6^{\mathrm{a}}$ & $3.8 \pm 0.5^{\mathrm{ab}}$ \\
\hline & 48 & $5.9 \pm 1.0^{c}$ & $5.1 \pm 2.9^{c}$ & $5.9 \pm 1.4^{\mathrm{c}}$ & $6.1 \pm 0.9^{c}$ \\
\hline & 72 & $3.3 \pm 0.4^{\mathrm{ab}}$ & $3.8 \pm 0.9^{b}$ & $4.2 \pm 0.6^{\mathrm{abc}}$ & $3.5 \pm 0.7^{\mathrm{ab}}$ \\
\hline & 96 & $2.3 \pm 0.6^{\mathrm{a}}$ & $4.0 \pm 1.3^{\mathrm{b}}$ & $5.7 \pm 1.5^{\mathrm{c}}$ & $2.8 \pm 0.4^{\mathrm{a}}$ \\
\hline & 120 & $2.5 \pm 1.6^{\mathrm{a}}$ & $4.4 \pm 0.7^{b}$ & $4.5 \pm 1.3^{b c}$ & $2.5 \pm 0.6^{\mathrm{a}}$ \\
\hline \multirow{6}{*}{$\begin{array}{c}\mathrm{Na}^{+} \\
(\mathrm{mEq} / \mathrm{L})\end{array}$} & 0 & $163.5 \pm 2.1^{\mathrm{ab}}$ & $162.5 \pm 3.5^{\mathrm{a}}$ & $166.0 \pm 5.7^{\mathrm{ab}}$ & $164.8 \pm 3.5^{\mathrm{ab}}$ \\
\hline & 24 & $171.7 \pm 3.5^{\mathrm{c}}$ & $173.3 \pm 4.9^{\mathrm{ab}}$ & $170.0 \pm 5.3^{\mathrm{bc}}$ & $173.9 \pm 4.0^{c}$ \\
\hline & 48 & $168.0 \pm 4.6^{\mathrm{bc}}$ & $168.7 \pm 3.8^{\mathrm{ab}}$ & $171.0 \pm 6.2^{\mathrm{bc}}$ & $169.1 \pm 3.1^{b c}$ \\
\hline & 72 & $166.3 \pm 2.5^{\mathrm{abc}}$ & $165.3 \pm 4.6^{\mathrm{ab}}$ & $166.7 \pm 4.9^{\mathrm{ab}}$ & $167.5 \pm 3.4^{\mathrm{abc}}$ \\
\hline & 96 & $161.0 \pm 2.0^{\mathrm{a}}$ & $169.3 \pm 2.9^{\mathrm{ab}}$ & $164.0 \pm 3.6^{\mathrm{a}}$ & $161.5 \pm 1.8^{\mathrm{a}}$ \\
\hline & 120 & $166.0 \pm 2.6^{\mathrm{abc}}$ & $166.3 \pm 3.5^{\mathrm{b}}$ & $168.3 \pm 2.3^{b}$ & $165.7 \pm 3.7^{a b c}$ \\
\hline \multirow{6}{*}{$\begin{array}{c}\mathrm{K}^{+} \\
(\mathrm{mEq} / \mathrm{L})\end{array}$} & 0 & $3.6 \pm 0.2^{b}$ & $3.7 \pm 0.4^{\mathrm{b}}$ & $3.9 \pm 0.2^{b}$ & $3.8 \pm 0.2^{b}$ \\
\hline & 24 & $3.3 \pm 0.3^{\mathrm{ab}}$ & $4.2 \pm 1.4^{\mathrm{bc}}$ & $4.1 \pm 1.3^{\mathrm{bc}}$ & $3.5 \pm 0.1^{\mathrm{ab}}$ \\
\hline & 48 & $3.3 \pm 0.2^{\mathrm{ab}}$ & $3.1 \pm 1.0^{\mathrm{a}}$ & $4.0 \pm 1.4^{\mathrm{bc}}$ & $3.4 \pm 0.3^{\mathrm{ab}}$ \\
\hline & 72 & $3.2 \pm 0.1^{\mathrm{ab}}$ & $2.9 \pm 0.2^{\mathrm{a}}$ & $2.9 \pm 0.2^{\mathrm{a}}$ & $3.3 \pm 0.2^{\mathrm{ab}}$ \\
\hline & 96 & $3.1 \pm 0.4^{\mathrm{ab}}$ & $3.1 \pm 0.3^{\mathrm{a}}$ & $3.1 \pm 0.3^{\mathrm{a}}$ & $3.2 \pm 0.3^{\mathrm{ab}}$ \\
\hline & 120 & $3.0 \pm 0.1^{\mathrm{a}}$ & $3.2 \pm 0.4^{\mathrm{a}}$ & $3.3 \pm 0.4^{\mathrm{a}}$ & $2.8 \pm 0.1^{\mathrm{a}}$ \\
\hline \multirow{6}{*}{$\begin{array}{c}\mathrm{Cl}^{-} \\
(\mathrm{mEq} / \mathrm{L})\end{array}$} & 0 & $135.5 \pm 0.7^{\mathrm{a}}$ & $137.5 \pm 3.5^{\mathrm{a}}$ & $139.0 \pm 4.2^{\mathrm{b}}$ & $131.4 \pm 1.9^{\mathrm{a}}$ \\
\hline & 24 & $141.0 \pm 2.0^{\mathrm{b}}$ & $145.0 \pm 3.5^{\mathrm{bc}}$ & $135.0 \pm 2.8^{\mathrm{a}}$ & $143.8 \pm 3.8^{\mathrm{b}}$ \\
\hline & 48 & $139.7 \pm 5.1^{\mathrm{ab}}$ & $139.3 \pm 2.3^{\mathrm{ab}}$ & $142.0 \pm 5.0^{\mathrm{c}}$ & $139.1 \pm 3.1^{\mathrm{ab}}$ \\
\hline & 72 & $138.3 \pm 1.2^{\mathrm{ab}}$ & $143.3 \pm 5.7^{\mathrm{b}}$ & $137.7 \pm 6.0^{\mathrm{ab}}$ & $137.7 \pm 2.2^{\mathrm{ab}}$ \\
\hline & 96 & $136.0 \pm 3.0^{\mathrm{ab}}$ & $144.3 \pm 3.5^{\mathrm{b}}$ & $137.3 \pm 3.1^{\mathrm{ab}}$ & $136.5 \pm 1.8^{a b}$ \\
\hline & 120 & $138.7 \pm 4.2^{\mathrm{ab}}$ & $137.7 \pm 2.5^{\mathrm{a}}$ & $140.0 \pm 1.0^{\mathrm{bc}}$ & $135.7 \pm 3.8^{\mathrm{ab}}$ \\
\hline
\end{tabular}


Table 2. Continued

\begin{tabular}{|c|c|c|c|c|c|}
\hline \multirow{2}{*}{ Items } & \multirow{2}{*}{$\begin{array}{c}\text { Elapsed time } \\
\text { (hrs) }\end{array}$} & \multicolumn{4}{|c|}{ Experiment groups } \\
\hline & & River puffer & Hybrids & Hybrid triploids & Tiger puffer \\
\hline \multirow{6}{*}{$\begin{array}{l}\text { Aspartate ami- } \\
\text { notransferase } \\
\text { (IU/L) }\end{array}$} & 0 & $04.0 \pm 2.8^{\mathrm{abb}}$ & $5.1 \pm 0.1^{\mathrm{ab}}$ & $5.4 \pm 0.9^{\mathrm{ab}}$ & $05.1 \pm 1.4^{\mathrm{abb}}$ \\
\hline & 24 & $05.5 \pm 0.7^{\text {bab }}$ & $3.3 \pm 2.1^{\mathrm{ab}}$ & $3.3 \pm 1.5^{\mathrm{ab}}$ & $15.5 \pm 2.1^{\mathrm{ccc}}$ \\
\hline & 48 & $09.0 \pm 5.7^{\text {caa }}$ & $8.0 \pm 1.4^{\mathrm{bb}}$ & $4.7 \pm 3.1^{\mathrm{ab}}$ & $011.5 \pm 4.9^{\mathrm{bcc}}$ \\
\hline & 72 & $05.5 \pm 2.1^{\mathrm{abb}}$ & $2.0 \pm 1.4^{\mathrm{ab}}$ & $4.0 \pm 2.6^{\mathrm{ab}}$ & $09.7 \pm 4.8^{\mathrm{abc}}$ \\
\hline & 96 & $03.5 \pm 4.9^{\mathrm{acb}}$ & $1.5 \pm 0.7^{\mathrm{ab}}$ & $2.5 \pm 2.1^{\mathrm{ab}}$ & $04.9 \pm 3.1^{\mathrm{abb}}$ \\
\hline & 120 & $02.3 \pm 0.6^{\mathrm{abb}}$ & $4.3 \pm 2.3^{\mathrm{ab}}$ & $6.0 \pm 3.6^{\mathrm{ab}}$ & $02.1 \pm 0.4^{\mathrm{abb}}$ \\
\hline \multirow{6}{*}{$\begin{array}{l}\text { Alanine aminotrans- } \\
\text { ferase } \\
\text { (IU/L) }\end{array}$} & 0 & $01.7 \pm 1.2^{\mathrm{abb}}$ & $2.2 \pm 0.2^{\mathrm{ab}}$ & $1.5 \pm 0.6^{\mathrm{ab}}$ & $01.8 \pm 0.7^{\mathrm{abb}}$ \\
\hline & 24 & $02.0 \pm 0.0^{\mathrm{abb}}$ & $1.7 \pm 1.2^{\mathrm{ab}}$ & $1.3 \pm 0.6^{\mathrm{ab}}$ & $02.1 \pm 0.1^{\mathrm{abb}}$ \\
\hline & 48 & $02.5 \pm 0.7^{\mathrm{abb}}$ & $2.5 \pm 0.7^{\mathrm{ab}}$ & $2.3 \pm 1.5^{\mathrm{ab}}$ & $03.2 \pm 0.6^{\mathrm{bbb}}$ \\
\hline & 72 & $01.7 \pm 1.2^{\mathrm{abb}}$ & $1.3 \pm 0.6^{\mathrm{ab}}$ & $2.7 \pm 1.2^{\mathrm{ab}}$ & $01.9 \pm 0.8^{\mathrm{abb}}$ \\
\hline & 96 & $01.7 \pm 0.2^{\mathrm{abb}}$ & $1.3 \pm 0.6^{\mathrm{ab}}$ & $2.0 \pm 1.4^{\mathrm{ab}}$ & $01.4 \pm 0.3^{\mathrm{abb}}$ \\
\hline & 120 & $01.3 \pm 0.6^{\mathrm{abb}}$ & $2.0 \pm 0.0^{\mathrm{ab}}$ & $2.7 \pm 1.5^{\mathrm{ab}}$ & $01.3 \pm 0.6^{\mathrm{abb}}$ \\
\hline
\end{tabular}

*Hybrids from river puffer $(+) \times$ tiger puffer $\left({ }^{\Uparrow}\right)$. The salinities of each group were changed from 30 psu to 0 psu. The values are mean $\pm \mathrm{SD}(\mathrm{n}=50)$ of triplicate experiments. Means within each column in item followed by the alphabetic letter are not significantly different $(p>0.05)$.

At $120 \mathrm{hrs}$, the red blood cells of the hybrids and hybrid triploids were lower than those of the other groups, and the red blood cells of the river puffer and tiger puffer were lower than those of their hybrids and hybrid triploids $(p<0.05)$. The trends for the plasma ions $\left(\mathrm{Na}^{+}, \mathrm{K}^{+}\right.$, and $\left.\mathrm{Cl}^{-}\right)$, aspartate aminotransferase (AST), and alanine aminotransferase (ALT) were similar to those for the red blood cell.

Table 3 shows blood and plasma factors during the hypo-osmoregulation of salinity. All blood and plasma factors for the river puffer, hybrids, hybrid triploids, and tiger puffer increased for 48, 72, or $96 \mathrm{hrs,} \mathrm{and} \mathrm{decreased}$ thereafter. However, the change in all blood and plasma factors during hyper-osmoregulation for $120 \mathrm{hrs}$ showed a trend opposite to that under hyper-osmoregulation. Significant differences in all blood and plasma factors among each species during hypo-osmoregulation were contrary to those during hyper-osmoregulation. For example, the hematocrit values of the river puffer, hybrids and hybrid triploids at $120 \mathrm{hrs}$ were lower than those at $0 \mathrm{hr}(p<0.05)$. The hematocrit values of the tiger puffer at $120 \mathrm{hrs}$ were higher than those at $0 \mathrm{hr}(p<0.05)$. At $0 \mathrm{hr}$, the hematocrit values of the tiger puffer were lower than those of the other groups, and the hematocrit values of the hybrid triploids were the highest $(p<0.05)$. At $120 \mathrm{hrs}$, the hematocrit values of the tiger puffer were higher than those of the other groups, and the hematocrit values of the hybrids were the lowest $(p<0.05)$.

\section{Stress response}

Fig. 1 shows the concentrations of plasma cortisol in the blood plasma of the river puffer, tiger puffer, hybrids, and hybrid triploids during hyper-osmoregulation A (30 $\mathrm{psu} \rightarrow 0 \mathrm{psu})$ and hyper-osmoregulation $\mathrm{B}(30 \mathrm{psu} \rightarrow 15$ psu). During hyper-osmoregulation A, the cortisol concentration of the river puffer, hybrids, hybrid triploids, and tiger puffer increased for $48 \mathrm{hrs}$, and decreased thereafter (Fig. 1a). The cortisol concentration of the tiger puffer was the highest during $168 \mathrm{hrs}$, and that of the hybrid triploids was the lowest $(p<0.05$; Fig. 1a). The cortisol concentration of the hybrids was between that of 
Table 3. Comparison of blood and plasma factors during $120 \mathrm{hrs}$ after hypo-osmoregulation of salinity in river puffer, Takifugu obscurus, tiger puffer, T. rubripes, hybrids (river puffer $\odot \times$ tiger puffer ${ }^{\lambda}$ ), and hybrid triploids*

\begin{tabular}{|c|c|c|c|c|c|}
\hline \multirow{2}{*}{ Items } & \multirow{2}{*}{$\begin{array}{c}\text { Elapsed time } \\
(\mathrm{hrs})\end{array}$} & \multicolumn{4}{|c|}{ Experiment groups } \\
\hline & & River puffer & Hybrids & Hybrid triploids & Tiger puffer \\
\hline \multirow{6}{*}{$\begin{array}{c}\text { Hematocrit } \\
(\%)\end{array}$} & 0 & $25.8 \pm 3.0^{\mathrm{a}}$ & $29.1 \pm 3.3^{\mathrm{ab}}$ & $36.6 \pm 3.4^{\mathrm{bc}}$ & $24.8 \pm 2.4^{\mathrm{a}}$ \\
\hline & 24 & $31.8 \pm 7.4^{\mathrm{a}}$ & $35.3 \pm 7.2^{\mathrm{ab}}$ & $40.7 \pm 9.7^{\mathrm{bc}}$ & $31.0 \pm 5.1^{\mathrm{ab}}$ \\
\hline & 48 & $31.0 \pm 5.6^{\mathrm{ab}}$ & $28.6 \pm 9.0^{\mathrm{a}}$ & $38.4 \pm 6.4^{\mathrm{b}}$ & $31.4 \pm 6.0^{\mathrm{ab}}$ \\
\hline & 72 & $38.9 \pm 7.4^{b}$ & $40.0 \pm 6.5^{\mathrm{b}}$ & $43.2 \pm 5.5^{\mathrm{c}}$ & $38.0 \pm 8.1^{\mathrm{b}}$ \\
\hline & 96 & $32.5 \pm 3.2^{\mathrm{ab}}$ & $41.8 \pm 8.6^{\mathrm{b}}$ & $24.1 \pm 7.2^{\mathrm{a}}$ & $35.5 \pm 3.9^{\mathrm{ab}}$ \\
\hline & 120 & $24.4 \pm 4.9^{\mathrm{a}}$ & $24.3 \pm 5.9^{\mathrm{a}}$ & $25.4 \pm 4.1^{\mathrm{b}}$ & $28.4 \pm 5.1^{\mathrm{a}}$ \\
\hline \multirow{6}{*}{$\begin{array}{l}\text { Hemoglobin } \\
(\mathrm{g} / \mathrm{dL})\end{array}$} & 0 & $12.4 \pm 0.7^{\mathrm{ab}}$ & $10.6 \pm 3.9^{\mathrm{a}}$ & $14.7 \pm 0.1^{b}$ & $11.0 \pm 1.1^{\mathrm{ab}}$ \\
\hline & 24 & $11.0 \pm 1.3^{\mathrm{ab}}$ & $13.0 \pm 1.3^{\mathrm{ab}}$ & $14.4 \pm 0.9^{b}$ & $12.4 \pm 1.5^{\mathrm{ab}}$ \\
\hline & 48 & $14.3 \pm 2.3^{b}$ & $14.9 \pm 3.4^{\mathrm{ab}}$ & $14.5 \pm 1.3^{\mathrm{b}}$ & $14.8 \pm 2.8^{b}$ \\
\hline & 72 & $14.5 \pm 2.3^{b}$ & $17.5 \pm 4.1^{b}$ & $15.8 \pm 0.6^{\mathrm{b}}$ & $15.5 \pm 3.1^{\mathrm{b}}$ \\
\hline & 96 & $12.8 \pm 0.8^{\mathrm{ab}}$ & $14.8 \pm 0.4^{\mathrm{ab}}$ & $11.1 \pm 3.0^{\mathrm{a}}$ & $12.1 \pm 0.9^{\mathrm{ab}}$ \\
\hline & 120 & $9.8 \pm 0.3^{\mathrm{a}}$ & $10.1 \pm 0.6^{\mathrm{a}}$ & $10.1 \pm 0.7^{\mathrm{a}}$ & $10.8 \pm 1.0^{\mathrm{a}}$ \\
\hline \multirow{6}{*}{$\begin{array}{l}\text { Red blood cell } \\
\left(\times 10^{6} \text { cell } / \mu \mathrm{L}\right)\end{array}$} & 0 & $4.5 \pm 1.6^{\mathrm{bc}}$ & $4.4 \pm 0.7^{b}$ & $4.5 \pm 1.3^{\mathrm{bc}}$ & $4.5 \pm 1.0^{\mathrm{bc}}$ \\
\hline & 24 & $2.3 \pm 0.6^{\mathrm{a}}$ & $4.0 \pm 1.3^{\mathrm{b}}$ & $5.7 \pm 1.5^{\mathrm{c}}$ & $2.8 \pm 0.4^{\mathrm{a}}$ \\
\hline & 48 & $3.3 \pm 0.4^{\mathrm{ab}}$ & $3.8 \pm 0.9^{b}$ & $4.2 \pm 0.6^{\mathrm{abc}}$ & $3.5 \pm 0.7^{\mathrm{ab}}$ \\
\hline & 72 & $5.9 \pm 1.0^{c}$ & $5.1 \pm 2.9^{c}$ & $5.9 \pm 1.4^{\mathrm{c}}$ & $6.1 \pm 0.9^{c}$ \\
\hline & 96 & $3.6 \pm 0.1^{\mathrm{ab}}$ & $4.3 \pm 0.3^{b}$ & $2.2 \pm 0.6^{\mathrm{a}}$ & $3.8 \pm 0.5^{\mathrm{ab}}$ \\
\hline & 120 & $2.7 \pm 0.7^{\mathrm{a}}$ & $2.8 \pm 0.5^{\mathrm{a}}$ & $2.6 \pm 0.5^{\mathrm{ab}}$ & $2.9 \pm 1.1^{\mathrm{a}}$ \\
\hline \multirow{6}{*}{$\begin{array}{c}\mathrm{Na}^{+} \\
(\mathrm{mEq} / \mathrm{L})\end{array}$} & 0 & $166.0 \pm 2.6^{\mathrm{abc}}$ & $166.3 \pm 3.5^{b}$ & $168.3 \pm 2.3^{b}$ & $165.7 \pm 3.7^{\mathrm{abc}}$ \\
\hline & 24 & $161.0 \pm 2.0^{\mathrm{a}}$ & $169.3 \pm 2.9^{\mathrm{ab}}$ & $164.0 \pm 3.6^{\mathrm{a}}$ & $161.5 \pm 1.8^{a}$ \\
\hline & 48 & $166.3 \pm 2.5^{\mathrm{abc}}$ & $165.3 \pm 4.6^{\mathrm{ab}}$ & $166.7 \pm 4.9^{\mathrm{ab}}$ & $167.5 \pm 3.4^{\mathrm{abc}}$ \\
\hline & 72 & $168.0 \pm 4.6^{\mathrm{bc}}$ & $168.7 \pm 3.8^{\mathrm{ab}}$ & $171.0 \pm 6.2^{\mathrm{bc}}$ & $169.1 \pm 3.1^{b c}$ \\
\hline & 96 & $171.7 \pm 3.5^{\mathrm{c}}$ & $173.3 \pm 4.9^{\mathrm{ab}}$ & $170.0 \pm 5.3^{\mathrm{bc}}$ & $173.9 \pm 4.0^{\mathrm{c}}$ \\
\hline & 120 & $163.5 \pm 2.1^{\mathrm{ab}}$ & $162.5 \pm 3.5^{\mathrm{a}}$ & $166.0 \pm 5.7^{\mathrm{ab}}$ & $164.8 \pm 3.5^{\mathrm{ab}}$ \\
\hline \multirow{6}{*}{$\begin{array}{c}\mathrm{K}^{+} \\
(\mathrm{mEq} / \mathrm{L})\end{array}$} & 0 & $3.0 \pm 0.1^{\mathrm{a}}$ & $3.2 \pm 0.4^{\mathrm{a}}$ & $3.3 \pm 0.4^{\mathrm{a}}$ & $2.8 \pm 0.1^{\mathrm{a}}$ \\
\hline & 24 & $3.1 \pm 0.4^{\mathrm{ab}}$ & $3.1 \pm 0.3^{\mathrm{a}}$ & $3.1 \pm 0.3^{\mathrm{a}}$ & $3.2 \pm 0.3^{\mathrm{ab}}$ \\
\hline & 48 & $3.2 \pm 0.1^{\mathrm{ab}}$ & $2.9 \pm 0.2^{\mathrm{a}}$ & $2.9 \pm 0.2^{\mathrm{a}}$ & $3.3 \pm 0.2^{\mathrm{ab}}$ \\
\hline & 72 & $3.3 \pm 0.2^{\mathrm{ab}}$ & $3.1 \pm 1.0^{\mathrm{a}}$ & $4.0 \pm 1.4^{\mathrm{bc}}$ & $3.4 \pm 0.3^{\mathrm{ab}}$ \\
\hline & 96 & $3.3 \pm 0.3^{\mathrm{ab}}$ & $4.2 \pm 1.4^{\mathrm{bc}}$ & $4.1 \pm 1.3^{\mathrm{bc}}$ & $3.5 \pm 0.1^{\mathrm{ab}}$ \\
\hline & 120 & $3.6 \pm 0.2^{\mathrm{b}}$ & $3.7 \pm 0.4^{\mathrm{b}}$ & $3.9 \pm 0.2^{\mathrm{b}}$ & $3.8 \pm 0.2^{\mathrm{b}}$ \\
\hline \multirow{6}{*}{$\begin{array}{c}\mathrm{Cl}^{-} \\
(\mathrm{mEq} / \mathrm{L})\end{array}$} & 0 & $138.7 \pm 4.2^{\mathrm{ab}}$ & $137.7 \pm 2.5^{\mathrm{a}}$ & $140.0 \pm 1.0^{\mathrm{bc}}$ & $135.7 \pm 3.8^{\mathrm{ab}}$ \\
\hline & 24 & $136.0 \pm 3.0^{\mathrm{ab}}$ & $144.3 \pm 3.5^{\mathrm{b}}$ & $137.3 \pm 3.1^{\mathrm{ab}}$ & $136.5 \pm 1.8^{a b}$ \\
\hline & 48 & $138.3 \pm 1.2^{\mathrm{ab}}$ & $143.3 \pm 5.7^{b}$ & $137.7 \pm 6.0^{\mathrm{ab}}$ & $137.7 \pm 2.2^{\mathrm{ab}}$ \\
\hline & 72 & $139.7 \pm 5.1^{\mathrm{ab}}$ & $139.3 \pm 2.3^{\mathrm{ab}}$ & $142.0 \pm 5.0^{\mathrm{c}}$ & $139.1 \pm 3.1^{\mathrm{ab}}$ \\
\hline & 96 & $141.0 \pm 2.0^{\mathrm{b}}$ & $145.0 \pm 3.5^{\mathrm{bc}}$ & $135.0 \pm 2.8^{\mathrm{a}}$ & $143.8 \pm 3.8^{\mathrm{b}}$ \\
\hline & 120 & $135.5 \pm 0.7^{\mathrm{a}}$ & $137.5 \pm 3.5^{\mathrm{a}}$ & $139.0 \pm 4.2^{\mathrm{b}}$ & $131.4 \pm 1.9^{\mathrm{a}}$ \\
\hline
\end{tabular}


Table 3. Continued

\begin{tabular}{|c|c|c|c|c|c|}
\hline \multirow{2}{*}{ Items } & \multirow{2}{*}{$\begin{array}{c}\text { Elapsed time } \\
\text { (hrs) }\end{array}$} & \multicolumn{4}{|c|}{ Experiment groups } \\
\hline & & River puffer & Hybrids & Hybrid triploids & Tiger puffer \\
\hline \multirow{6}{*}{$\begin{array}{l}\text { Aspartate ami- } \\
\text { notransferase } \\
\text { (IU/L) }\end{array}$} & 0 & $2.3 \pm 0.6^{\mathrm{a}}$ & $4.3 \pm 2.3^{\mathrm{ab}}$ & $6.0 \pm 3.6^{\mathrm{ab}}$ & $2.1 \pm 0.4^{\mathrm{a}}$ \\
\hline & 24 & $3.5 \pm 4.9^{\mathrm{ac}}$ & $1.5 \pm 0.7^{\mathrm{a}}$ & $2.5 \pm 2.1^{\mathrm{a}}$ & $4.9 \pm 3.1^{\mathrm{ab}}$ \\
\hline & 48 & $5.5 \pm 2.1^{\mathrm{ab}}$ & $2.0 \pm 1.4^{\mathrm{a}}$ & $4.0 \pm 2.6^{\mathrm{ab}}$ & $9.7 \pm 4.8^{\mathrm{abc}}$ \\
\hline & 72 & $9.0 \pm 5.7^{\mathrm{c}}$ & $8.0 \pm 1.4^{b}$ & $4.7 \pm 3.1^{\mathrm{ab}}$ & $11.5 \pm 4.9^{\mathrm{bc}}$ \\
\hline & 96 & $5.5 \pm 0.7^{b}$ & $3.3 \pm 2.1^{\mathrm{a}}$ & $3.3 \pm 1.5^{\mathrm{ab}}$ & $15.5 \pm 2.1^{\mathrm{c}}$ \\
\hline & 120 & $4.0 \pm 2.8^{\mathrm{ab}}$ & $5.1 \pm 0.1^{\mathrm{ab}}$ & $5.4 \pm 0.9^{\mathrm{ab}}$ & $5.1 \pm 1.4^{\mathrm{ab}}$ \\
\hline \multirow{6}{*}{$\begin{array}{c}\text { Alanine ami- } \\
\text { notransferase } \\
\text { (IU/L) }\end{array}$} & 0 & $1.3 \pm 0.6^{\mathrm{a}}$ & $2.0 \pm 0.0^{\mathrm{ab}}$ & $2.7 \pm 1.5^{\mathrm{ab}}$ & $1.3 \pm 0.6^{\mathrm{a}}$ \\
\hline & 24 & $1.7 \pm 0.2^{\mathrm{a}}$ & $1.3 \pm 0.6^{\mathrm{a}}$ & $2.0 \pm 1.4^{\mathrm{ab}}$ & $1.4 \pm 0.3^{\mathrm{a}}$ \\
\hline & 48 & $1.7 \pm 1.2^{\mathrm{a}}$ & $1.3 \pm 0.6^{\mathrm{a}}$ & $2.7 \pm 1.2^{\mathrm{ab}}$ & $1.9 \pm 0.8^{\mathrm{a}}$ \\
\hline & 72 & $2.5 \pm 0.7^{\mathrm{ab}}$ & $2.5 \pm 0.7^{\mathrm{ab}}$ & $2.3 \pm 1.5^{\mathrm{ab}}$ & $3.2 \pm 0.6^{\mathrm{b}}$ \\
\hline & 96 & $2.0 \pm 0.0^{\mathrm{ab}}$ & $1.7 \pm 1.2^{\mathrm{a}}$ & $1.3 \pm 0.6^{\mathrm{a}}$ & $2.1 \pm 0.1^{\mathrm{ab}}$ \\
\hline & 120 & $1.7 \pm 1.2^{\mathrm{a}}$ & $2.2 \pm 0.2^{\mathrm{ab}}$ & $1.5 \pm 0.6^{\mathrm{a}}$ & $1.8 \pm 0.7^{\mathrm{a}}$ \\
\hline
\end{tabular}

*Hybrids from river puffer $(+) \times$ tiger puffer $\left({ }^{\pi}\right)$. The salinities of each group were changed from 0 psu to 30 psu. The values are mean $\pm \mathrm{SD}(\mathrm{n}=50)$ of triplicate experiments. Means within each column in item followed by the alphabetic letter are not significantly different $(p>0.05)$.

the river puffer and the tiger puffer, and was higher than that of the hybrid triploids ( $p<0.05$; Fig. 1a). During hyperosmoregulation $\mathrm{B}$, the cortisol concentration of the river puffer, hybrids, and hybrid triploids increased for $24 \mathrm{hrs,}$ and decreased thereafter. The cortisol concentration of the tiger puffer was elevated for $72 \mathrm{hrs,}$, and decreased thereafter (Fig. 1b). The cortisol concentration of the tiger puffer was the highest during $168 \mathrm{hrs}$, and that of the river puffer was the lowest ( $p<0.05$; Fig. 1b). The cortisol concentration of the hybrids was between that of the river puffer and the tiger puffer, and was higher than that of the hybrid triploids ( $p<0.05$; Fig. 1b).

As shown in Fig. 2, finding shows the concentrations of plasma cortisol in the blood plasma of the river puffer, tiger puffer, hybrids, and hybrid triploids during hypoosmoregulation A ( $0 \mathrm{psu} \rightarrow 30 \mathrm{psu})$ and hypo-osmoregulation B (0 psu $\rightarrow 30 \mathrm{psu})$. The cortisol concentrations of each species during hypo-osmoregulation exhibited faster recovery than during hyper-osmoregulation. During hypoosmoregulation $\mathrm{A}$, the cortisol concentrations of the hy- brids, hybrid triploids, and tiger puffer were elevated for 6 hrs, and decreased thereafter (Fig. 2a). The cortisol concentration of the river puffer was elevated for $12 \mathrm{hrs,}$ and decreased thereafter (Fig. 2a). The cortisol concentration of the tiger puffer was the highest in the first $2 \mathrm{hrs,}$ and the cortisol concentration of the river puffer was the highest from 6 to 48 hrs. The cortisol concentration of the hybrid triploids was the lowest ( $p<0.05$; Fig. 2a, b). For 48 hrs, the cortisol concentration of the hybrids was between that of the river puffer and the tiger puffer, and was higher than that of the hybrid triploids $(p<0.05$; Fig. 2a). During hyper-osmoregulation $\mathrm{B}$, the cortisol concentrations of the river puffer, hybrids, hybrid triploids, and tiger puffer were elevated for 6 hrs, and decreased thereafter (Fig. 2b). The cortisol concentration of the river puffer was the highest for $48 \mathrm{hrs}$, and the cortisol of the hybrid triploids was the lowest ( $p<0.05$; Fig. 2b). The cortisol concentration of the hybrids was lower than that of the river puffer and tiger puffer for $12 \mathrm{hrs}$. At 24 and $48 \mathrm{hrs}$, the cortisol concentration of the hybrids was between that of the river 

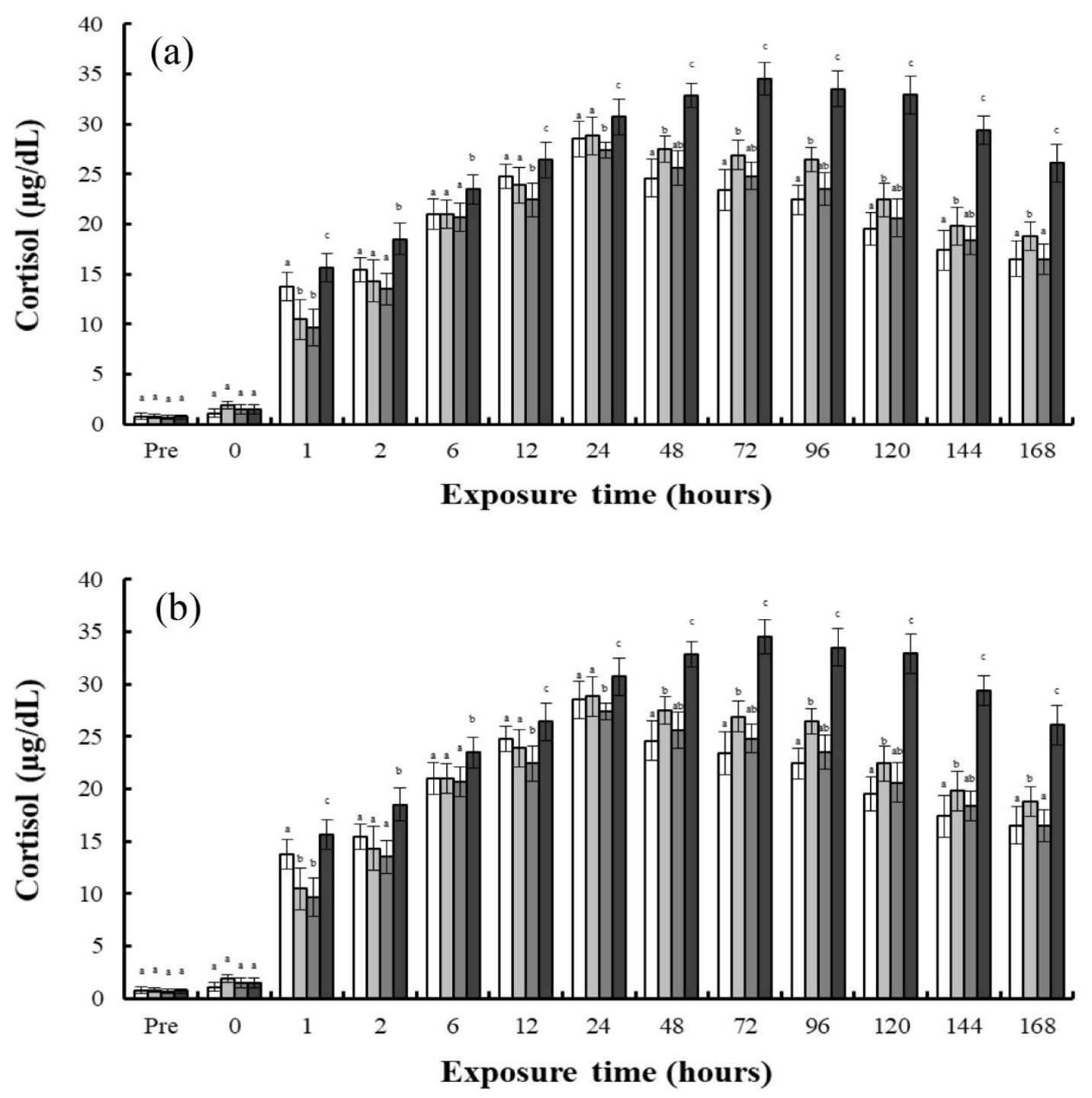

Fig. 1. Variations in the plasma cortisol concentrations in the blood plasma of river puffer, Takifugu obscures ( $\square$ ), tiger puffer, T. rubripes $\left(\square\right.$ ), hybrids (river puffer + xtiger puffer $\left.{ }^{3}\right)(\square$ ), and hybrid triploids $(\square)$, during 7 days after hyper-osmoregulation of salinity. (a): $30 \mathrm{psu} \rightarrow 0 \mathrm{psu}$; (b): $30 \mathrm{psu} \rightarrow 15 \mathrm{psu}$. Values are means $\pm \mathrm{SE}$ $(\mathrm{n}=50)$. Actually $\mathrm{n}=50$ for each experiment because the means and SE were calculated separately for each group. Different capital letters on error bars are significantly different for each group in same exposure time $(p<0.05)$. Different small letters on error bars are significantly different for each time in same experimental group ( $p<0.05)$. 'Pre' means control group before hypo-osmoregulation.

puffer and tiger puffer, and was higher than that of the hybrid triploids at $48 \mathrm{hrs}(p<0.05$; Fig. $2 \mathrm{~b})$.

\section{Histological response}

Fig. 3 shows the histological section for the gill filaments in each species. The chloride cells in the gill filaments of each species increased with increasing salinity. At 0 psu, the gill filament of the river puffer, hybrids, and hybrid triploids had fewer chloride cells than those of the tiger puffer (Fig. 3a, 3b, and 3c), and chloride cells were observed in the lamellae of the tiger puffer (Fig. 3d). At 30 psu, chloride cells were highest among the gill lamellae of the river puffer, hybrids, and hybrid triploids (Fig. 3a, 3b, and $3 \mathrm{c}$ ). In the gill filaments of the tiger puffer, the 

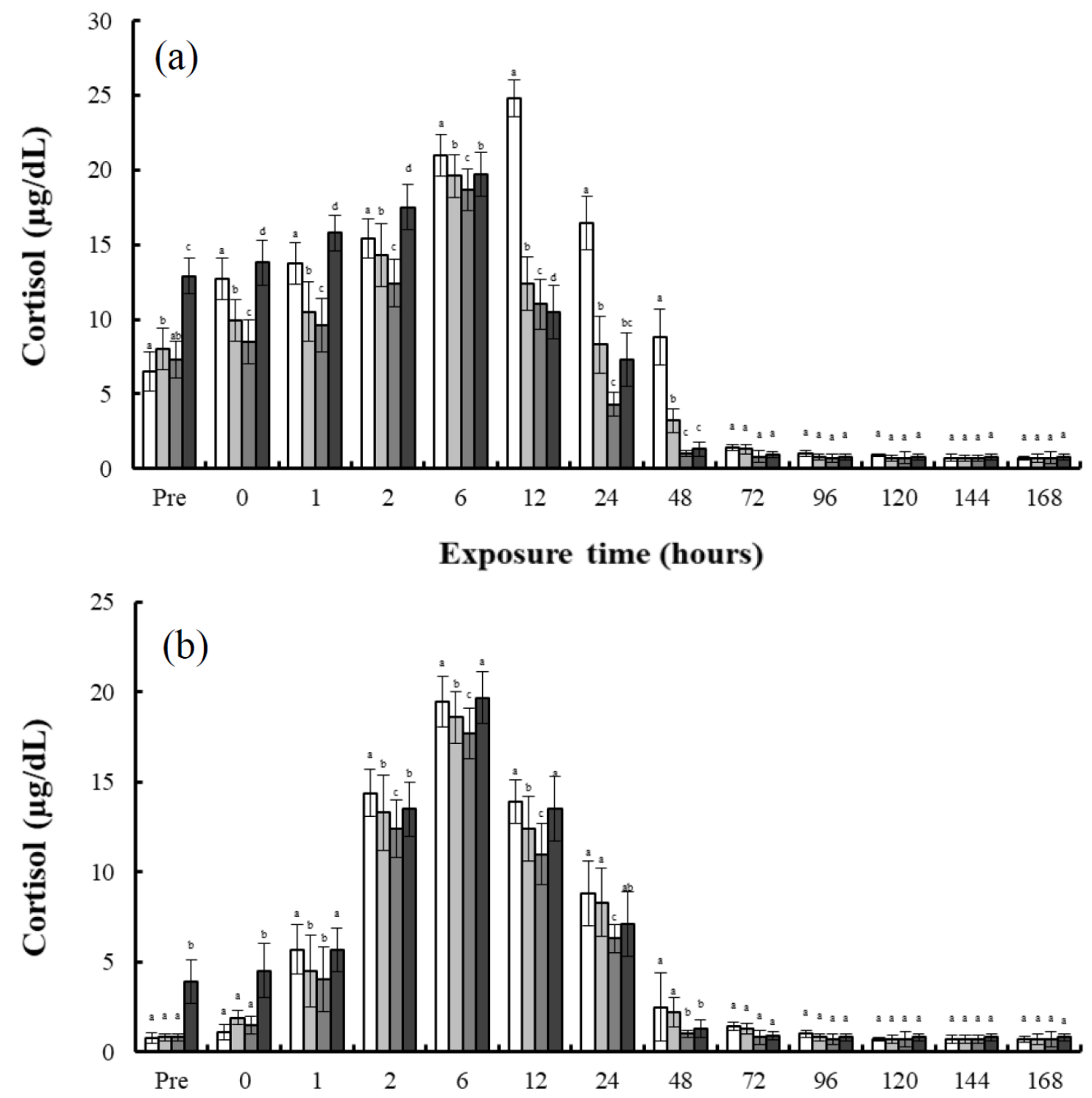

\section{Exposure time (hours)}

Fig. 2. Variations in the plasma cortisol concentrations in the blood plasma of river puffer, Takifugu obscures ( $\square$ ),

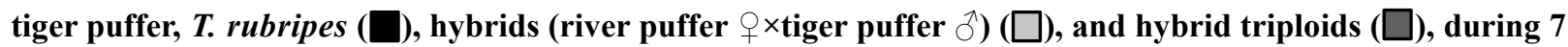
days after hypo-osmoregulation of salinity. (a): $0 \mathrm{psu} \rightarrow 30 \mathrm{psu}$; (b): $15 \mathrm{psu} \rightarrow 30 \mathrm{psu}$. Before experiment started, the salinities of each group were maintained 0 psu and 15 psu during 14 days, respectively. Values are means \pm SE $(n=50)$. Actually $n=50$ for each experiment because the means and SE were calculated separately for each group. Different capital letters on error bars are significantly different for each group in same exposure time $(p<0.05)$. Different small letters on error bars are significantly different for each time in same experimental group $(p<0.05)$. 'Pre' means control group before hypo-osmoregulation.

chloride cells were increased not only among the gill lamellae but also among the lamellae, and were observed throughout the lamellae (Fig. 3d). Fig. 4 shows histological sections of the kidneys in each species. Melano-macrophages (MMs) were increased with decreasing salinity (Fig. 4). At $30 \mathrm{psu}$, MMs were not observed in the kidney of each 


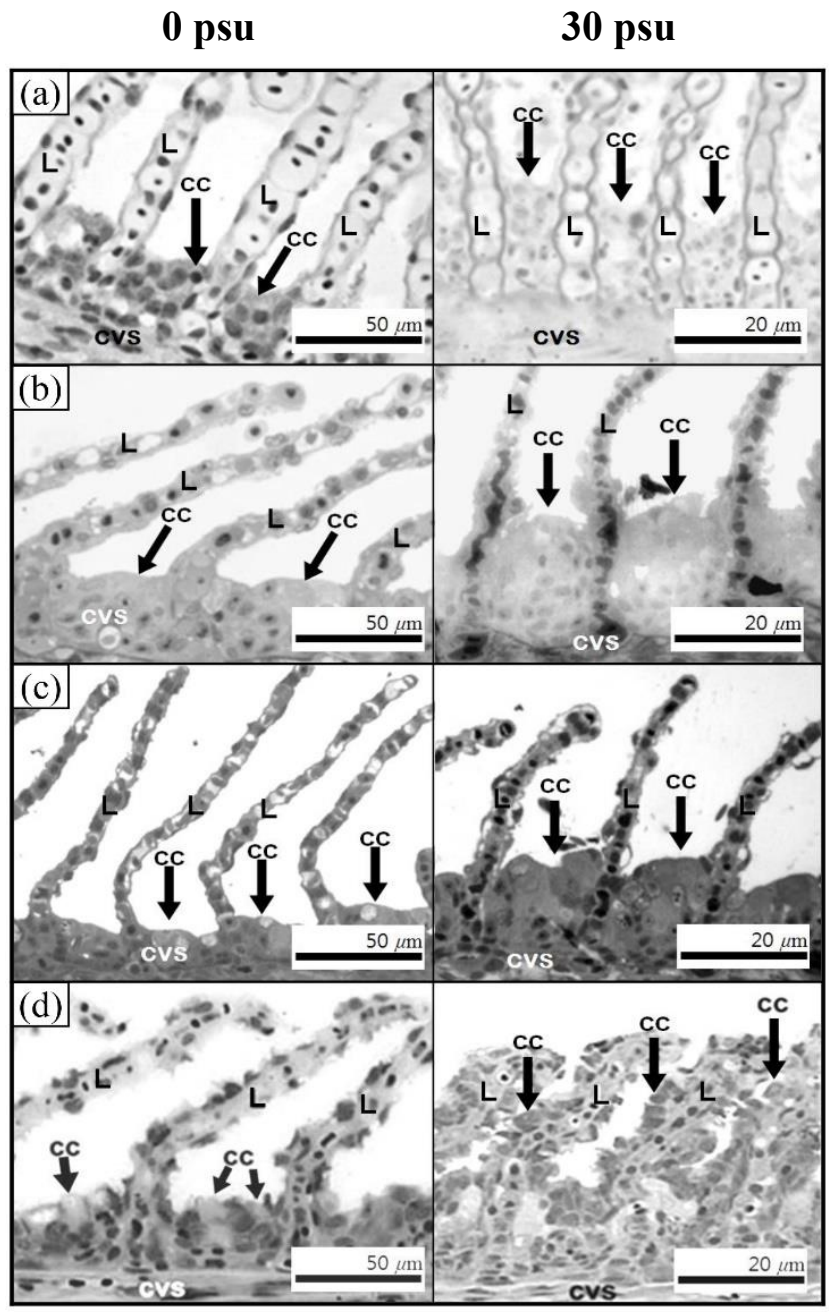

Fig. 3. Histological section of gill filaments viewed by light microscope in samples exposed to 0 psu (left side) and $30 \mathrm{psu}$ (right side) during $120 \mathrm{hrs}$ (a): river puffer, Takifugu obscurus; (b): hybrids (river puffer $Q \times$ tiger puffer $\widehat{\delta}$ ); (c): hybrid triploids; (d): tiger puffer, T. rubripes. cc: chloride cell (arrow); cvs: central venous sinus; L: lamellae.

species, but were observed at 0 psu. MMs in the kidney of the river puffer were the largest, and MMs in the kidney of the hybrid triploids were the smallest (Fig. 4a and 4c). MMs in the kidney of the hybrids were larger than those of hybrid triploids (Fig. 4b and 4c).

\section{DISCUSSIONS}

In river puffer, Takifugu obscurus, tiger puffer, $T$.

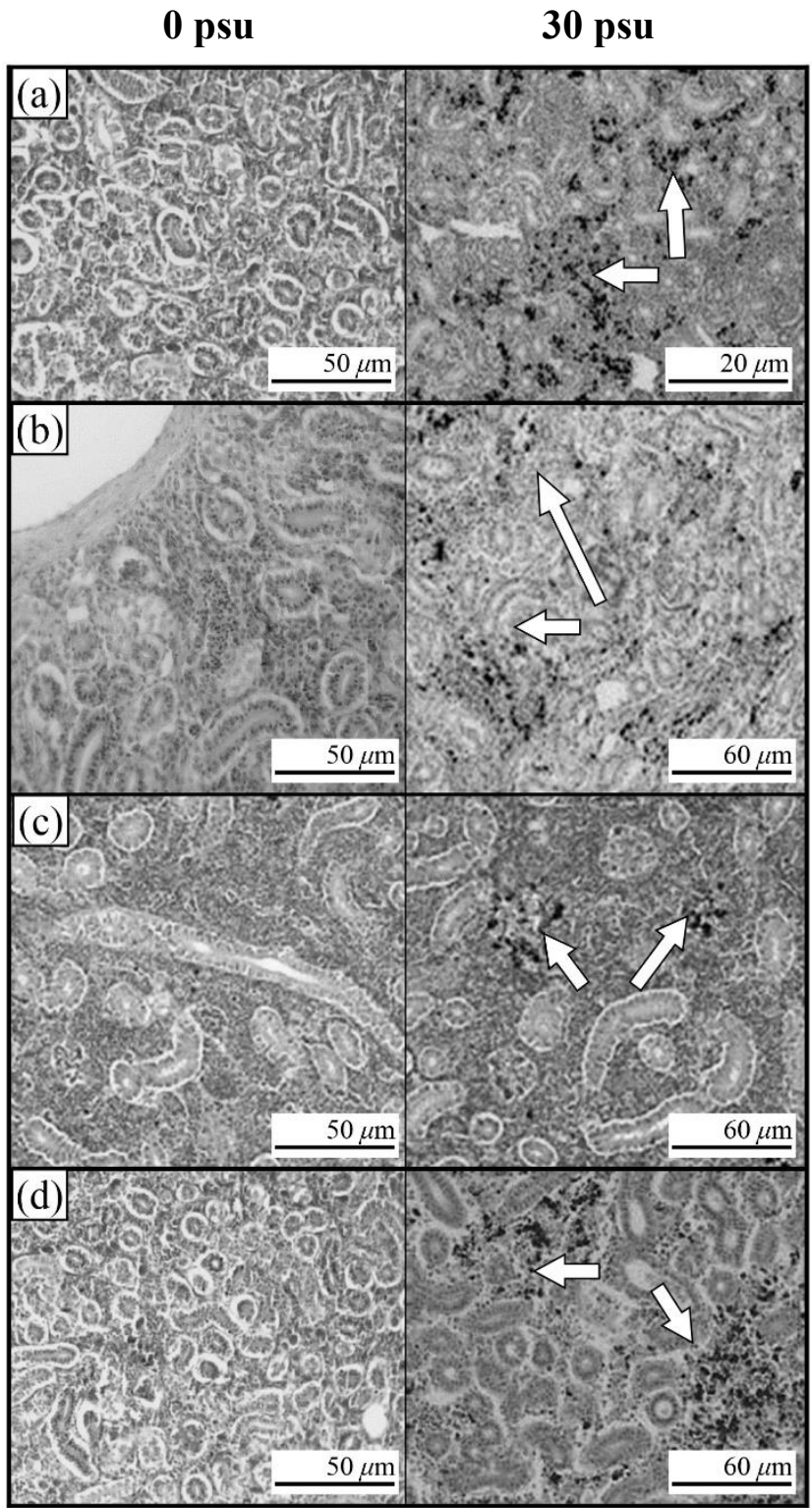

Fig. 4. Histological section of kidney secondary tubules viewed by light microscope in samples exposed to 0 psu (left side) and 30 psu (right side) during 120 hrs. (a): river puffer, Takifugu obscurus; (b): hybrids (river puffer $q \times$ tiger puffer $\left.{ }^{\Uparrow}\right)$ ); (c): hybrid triploids; (d): tiger puffer, T. rubripes. white arrows are MMs.

rubripes, their hybrids, and hybrid triploids, physiological responses of hypo-osmoregulation were more stable than those of hyper-osmoregulation. Cho et al. (2010) researched that survival rate in hyper-osmoregulation groups was higher than that in hypo-osmoregulation groups in marine 
medaka, Oryzias dancena. Marine medaka inhabits brackish water, but river puffer and tiger puffer inhabit seawater (Kang et al., 2007). So, our results may be contrary to the results of Cho et al. (2010).

In our study, hematocrit values of each species decreased with changing salinity. A similar trend of decreased hematocrit value with increasing salinity was observed in chinook salmon, Oncorhynchus tshawytscha fry (Morgan \& Iwama, 1991) and in sturgeon, Acipenser oxyrinehus (Altinok et al., 1998) when animals were transferred from diluted to full-strength seawater. On the other hand, the opposite phenomenon occurred in rainbow trout, $O$. mykiss (Morgan \& lwama, 1991). Morgan \& lwama (1991) attributed these variable responses to species-specific differences in red blood cell and plasma volume changes. Plasma ions $\left(\mathrm{Na}^{+}, \mathrm{K}^{+}\right.$, and $\left.\mathrm{Cl}^{-}\right)$increased and decreased with changing salinity, and plasma ions returned to basal levels within 1 week in this study. As mentioned Cech et al. (1996), plasma $\mathrm{Cl}^{-}$did not change until 2-4 hrs postexercise, when decreases (in fresh water and brackish water), increase (in sea water), or no change (in brackish water) indicated passive fluid or $\mathrm{Cl}^{-}$exchanges with the recovery environment in sub-adults of striped bass, Morone saxatilis. Higher $\mathrm{Na}^{+}$or $\mathrm{Cl}^{-}$with changing salinity was also observed in other species when individuals were exposed to different dilutions of seawater (Morgan \& lwama, 1991; Altinok et al., 1998; Vonck et al., 1998).

In this study, plasma cortisol also varied markedly but values were somewhat lower and more stable at $30 \mathrm{psu}$ in each species, respectively, compared to 0 psu and 15 psu. Lower cortisol levels might suggest either a natural preference for these particular salinity levels, resulting in decreased cortisol secretion, or an increase in the metabolic clearance rate of cortisol, as observed by Nichols \& Weisbart (1985) after transfer of Atlantic salmon, Salmo salar to seawater. Barton \& Iwama (1991) stated that "Usually, phenomenon that plasma cortisol concentration of fishes rises by stress is first order reaction, phenomenon that plasma glucose concentration rises is result of secondorder first order reaction by hormone rise reaction by stress". This trend has been reported in the gray mullet, Mugil cephalus and kelp grouper, Epinephelus bruneus (Barton \& Iwama, 1991; Park et al., 2008). Das et al. (2004) suggested that the greater use of glucose for increased cell metabolism during early exposure may have overwhelmed the increase in blood glucose, even though glycogenolysis would have increased during this period (Martinez-Alvarez et al., 2002). However, because of dysfunctional cell metabolism, the lower use of glucose later in the exposure period (after $48 \mathrm{hrs}$ ) resulted in an increase in blood glucose levels.

In this study, kidney of each groups appeared melanomacrophages (MMs) after decreasing salinity. Under the optical microscope, MM appear as small-to-large round or oval structures, which is definitely distinguished from the surrounding lymphatic tissue, whereas under the electron microscope, they appears in the form of groups of macrophages (Hur et al., 2006). MMs are observed in normal fish, but are more numerous in physiologically abnormal states such as disease or stress. Moreover, in this state, the number, size and shape of MMs vary (Hur et al., 2006). The cytoplasm of MM contains abundant melanin, hemosoderin, lipofuscine (fat-free lipochrome), and ceroid, which react positively to PAS and Ziehl-Neelson reactions. MM vary in colour from yellow to black, mainly because of tissue catabolism (Micale \& Perdichizzi, 1990). The colour intensity depends on the type of fish, its age and health status (Hur et al., 2006). Increased number of MMs are related to detoxification and destruction in response to endogenous or exogenous toxins, inflammatory response, stress response and circulation (Hur et al., 2006). MMs increased in the kidney of some teleosts, such as rainbow trout, during starvation (Hur et al., 2006). So, decreasing salinity was stress factor in river puffer, tiger puffer, their hybrids, and hybrid triploids.

Hematological response of hybrids was lower than that 
of hybrid triploids, and cortisol concentration of hybrids was higher than those of hybrid triploids during $120 \mathrm{hrs}$. Previous studies have different or similar trend compared to this study. Seol et al. (2008) compared hematological features pertaining to aerobic capability between diploids and triploids Far Eastern catfish, Silurus asotus. No significant differences between diploids and triploids were found for the haematocrit value, total haemoglobin, and mean corpuscular haemoglobin concentration, while the mean corpuscular volume, mean corpuscular haemoglobin, and plasma glucose concentration were significantly higher in triploids than in diploids, and the number of red blood cells was significantly lower in triploids than in diploids (Seol et al., 2008). The only controlled experiment to have assessed physiological aspects of the stress response of triploids is that of Biron \& Benfey (1994), who found no difference between triploids and diploids in hematocrit and plasma cortisol and glucose profiles after an acute handling stress. Poorer survival due to chronic stress may be reflected in reduced energy stores and/or increased rates of depletion of these stores during stressful conditions. Although substrate utilization during aerobic metabolism does not differ between triploids and diploids, it may differ in the ability of triploids to withstand sustained anaerobic metabolism (Ojolick et al., 1995). But the hormonal responses of cortisol and glucose in this study were different from previous study. In this study, plasma cortisol, glucose, and lactic acid of diploids were higher than triploids. The result of this study suggests that stress response between hybrid triploids and hybrids in plasma cortisol after water salinity stress differs. Stress response of hybrids was more sensitive than hybrid triploids.

Hematological response and stress response of hybrids showed middle values between river puffer and tiger puffer. Previous study has different or similar trend compared to this study. Lim et al. (2005) reported physiological responses of hybrids striped bass (Morone chrysops $\times$ M. saxatilis) to gradual changes of salinity from $33 \mathrm{psu}$ to 0 psu and from 0 psu to 33 psu in two separate trials. Gradual salinity change was not accompanied by an increase in cortisol level in the plasma, however, the glucose level in the plasma increased (Lim et al., 2005). $\mathrm{Na}^{+}, \mathrm{Cl}^{-}$and osmolality significantly increased with rising salinity, but they did not change with dropping salinity (Lim et al., 2005). The hybrid striped bass adjusted effectively without stress to the gentle change of salinity, and hybrid striped bass has middle values between their parents (Lim et al., 2005). The results of this experiment confirmed physiological response of hybrid triploids and hybrids between river puffer and tiger puffer. This experiment will be used as basic data for determining characteristics of hybrids and hybrid triploids.

\section{CONFLICT OF INTERESTS}

The authors declare no potential conflict of interest.

\section{ACKNOWLEDGEMENTS}

We are grateful to the staff of the Fishery Genetics and Breeding Sciences Laboratory of the Korea Maritime \& Ocean University (KMOU), Korea. The comments of anonymous reviewers greatly improved the quality of this manuscript. We declare that all the experiments in this study complied with the current laws of Korea (Ordinance of Agriculture, Food and Fisheries, No. 1) and the Ethical Guidelines of KMOU, Korea.

\section{REFERENCES}

Altinok I, Galli SM, Chapman FA (1998) Ionic and osmotic regulation capabilities of juvenile Gulf of Mexico sturgeon, Acipenser oxyrinchus. Comp Biochem Phys A 120:609-616.

Barton BA, Iwama GK (1991) Physiological changes in 
fish from stress in aquaculture with emphasis on the response and effects of corticosteroids. Annu Rev Fish Dis $1: 3-26$.

Benfey TJ (1999) The physiology and behavior of triploid fishes. Fish Sci 7:39-67.

Biron M, Benfey TJ (1994) Cortisol, glucose and hematocrit changes during acute stress, cohort sampling, and the diel cycle in diploid and triploid brook trout (Salvelinus fontinalis Mitchill). Fish Physiol Biochem 13:153-160.

Bryden CA, Heath JW, Heath DD (2004) Performance and heterosis in farmed and wild chinook salmon (Oncorhynchus tshawytscha) hybrid and purebred crosses. Aquaculture 235:249-261.

Cech JJ Jr, Bartholow SD, Young PS, Hopkins TE (1996) Striped bass exercise and handling stress in freshwater: Physiological responses to recovery environment. Trans Am Fish Soc 125:308-320.

Chevassus B (1983) Hybridization in fish. Aquaculture $33: 245-262$.

Cho YS, Lee SY, Kim DS, Nam YK (2010) Tolerance capacity to salinity changes in adult and larva of Oryzias dancena, a euryhaline medaka. Korean J Ichthyol 22:916.

Das PC, Ayyappan S, Jena JK, Das BK (2004) Nitrite toxicity in Cirrhinus mrigala (Ham): acute toxicity and sub-lethal effect on selected haematological parameters. Aquaculture 235:633-644.

Duncan DB (1955) Multiple range and multiple F tests. Biometrics 11:1-42.

Hur JW, Woo SR, Jo JH, Park IS (2006) Effects of starvation on kidney melano-macrophage centre in olive flounder, Paralichthys olivaceus (Temminck and Schlegel). Aquacult Res 37:821-825.

Jeong DY, Kim DS, Lee MJ, Kim SR, Byun DS, Kim HD, Park YH (1994) Toxicity of several puffers collected at a fish market of Pusan, Korea. Bull Korean Fish Soc 27:682-689.
Kang HW, Shim KB, Kang DY, Jo KC, Song KC, Lee JH, Song HI, Son SG, Cho YJ (2007) Sitological quality evaluation of cultured and wild river puffer, Takifugu obscurus (Abe). J Aquaculture 20:147-153.

Kikuchi K, Furuta T, Ishizuka H, Yanagawa T (2007) Growth of tiger puffer, Takifugu rubripes, at different salinities. J World Aquacult Soc 38:427-434.

Kim DS, Jo JY, Lee TY (1994) Induction of triploidy in mud loach (Misgurnus mizolepis) and its effect on gonad development and growth. Aquaculture 120:263270 .

Kim DS, Nam YK, Park IS (1995) Survival and karyological analysis of reciprocal diploid and triploid hybrids between mud loach (Misgurnus mizolepis) and cyprinid loach (Misgurnus anguillicaudatus). Aquaculture $135: 257-265$.

Kim JH, Son KT, Mok JS, Oh EG, Kim JK, Lee TK (2006) Toxicity of the puffer fish Takifugu porphyreus and Takifugu rubripes from coastal areas of Korea. J Korean Fish Soc 39:447-453.

Lim HK, Han HK, Lee JH, Jeong MH, Hur JW (2005) Effects of gradual change of salinity on physiological response in hybrid striped bass (Morone chrysops $\times M$. saxatilis). Korean J Ichthyol 17:43-48.

Martinez-Alvarez RM, Hidalgo MC, Domezain A, Morales AE, Grarcia-Gallego M, Sanz A (2002) Physiological changes of sturgeon Acipenser naccarii caused by increasing environmental salinity. J Exp Biol 205:36993706.

Micale V, Perdichizzi F (1990) A quantitative and histochemical study on melano-macrophage centres in the spleen of the teleost fish Diplodus annularis L. J Fish Biol 37:191-197.

Morgan JD, Iwama GK (1991) Effects of salinity on growth, metabolism, and ion regulation in juvenile rainbow and steelhead trout (Oncorhynchus mykiss) and fall chinook salmon (O. tshawytscha). Can J Fisher Aqua Sci 48:2083-2094 
Nichols DJ, Weisbart M (1985) Cortisol dynamics during seawater adaptation of Atlantic salmon Salmo salar. Am J Phys 248:R651-R659.

Ojolick EJ, Cusack R, Benfey TJ, Kerr SR (1995) Survival and growth of all-female diploid and triploid rainbow trout (Oncorhynchus mykiss) reared at chronic high temperature. Aquaculture 131:177-187.

Park IS (2019) Anaesthetic efficacy and physiological response of clove oil and lidocaine- $\mathrm{HCl}$ on river puffer, Takifugu obscurus and tiger puffer, T. rubripes. Dev Reprod 23:21-34.

Park IS, Kim CH, Choi GC, Kim DS (1997) Production of hybrid and allotriploid between rainbow trout, $\mathrm{On}$ corhynchus mykiss and cherry salmon, O. masou. I. Cytogenetic study. J Aquac 10:39-47.

Park IS, Kim DS (2000) Comparison of some tissues in diploid and triploid hybrid between mud loach, Misgurnus mizolepis and cyprinid loach, M. anguillicaudatus. Dev Reprod 4:19-28.

Park IS, Lim SY, Lee TH, Gil HW, Yoo GY (2017) Various characteristics of hybrid between river puffer, Takifugu obscures and tiger puffer, T. rubripes, and their hybrid triploid. Dev Reprod 21:181-191.

Park MO, Hur WJ, Im SY, Seol DW, Lee JH, Park IS (2008) Anaesthetic efficacy and physiological responses to clove oil-anaestheized kelp grouper Epinephelus bruneus. Aquacult Res 39:877-884.

Peruzzi S, Chatain B, Saillant E, Haffray P, Menu B, Falguie're J-C (2004) Production of meiotic gynogenetic and triploidsea bass, Dicentrarchus labrax L. 1. Performances, maturation and carcass quality. Aquaculture 230:41-64.

Rahman MA, Uehara T, Lawrence JM (2005) Growth and heterosis of hybrids of two closely related species of Pacific sea urchins (Genus echinometra) in Okinawa. Aquaculture 245:121-133.

Seol DW, Im SY, Hur WJ, Park MO, Kim DS, Jo JY, Park IS (2008) Haematological parameters and respiratory function in diploid and triploid Far Eastern catfish, $\mathrm{Si}$ lurus asotus. Genes Genom 30:205-213.

Vonck AP, Bonga SEW, Flik G (1998) Sodium and calcium balance in Mozambique tilapia, Oreochromis mossambicus, raised at different salinities. Comp Biochem Physiol A 119:441-449.

Yoo GW, Bai SC (2014) Effects of different dietary protein sources on apparent digestibility and growth in juvenile river puffer, Takifugu obscurus. Korean J Fish Aquat Soc 47:383-389.

Yoo GY, Lee TH, Gil HW, Lim SG, Park IS (2018) Cytogenetic analysis of hybrids and hybrid triploids between the river puffer, Takifugu obscurus and tiger puffer, T. rubripes. Aquacult Res 49:637-650. 